\title{
A CLASSIFICATION METHODOLOGY FOR COLOR TEXTURES USING MULTISPECTRAL RANDOM FIELD MATHEMATICAL MODELS
}

\author{
Alireza Khotanzad and Jesse W. Bennett \\ \{kha, jesse\}@engr.smu.edu \\ Electrical Engineering \\ Southern Methodist University \\ Dallas, Texas 75275-0338, USA
}

\author{
Orlando J. Hernandez \\ hernande@tcnj.edu \\ Electrical and Computer Engineering \\ The College of New Jersey \\ Ewing, New Jersey 08628-0718, USA
}

\begin{abstract}
A large number of texture classification approaches have been developed in the past but most of these studies target gray-level textures. In this paper, supervised classification of color textures is considered. Several different Multispectral Random Field models are used to characterize the texture. The classifying features are based on the estimated parameters of these model and functions defined on them. The approach is tested on a database of sixteen different color textures. A near $100 \%$ classification accuracy is achieved. The advantage of utilizing color information is demonstrated by converting color textures to gray-level ones and classifying them using gray-level random field models. It is shown that color based classification is significantly more accurate than its gray-level counterpart.
\end{abstract}

Keywords- Color Texture, Color Texture Features, Mutispectral Random Field Models, Texture Classification

\section{INTRODUCTION}

Texture classification has received a great deal of attention in the past and a large body of literature exists on it. However, a large majority of previously published studies on this subject consider classification of gray-level textures. Although color is an important visual cue, classification of color textures has not received much attention in the past since simultaneous consideration of textural activity and color information creates a higher degree of complexity. In this work, we focus on color texture classification and utilize a number of recently developed multispectral random field models for this purpose. This class of models is capable of characterizing color texture and as such provides the right tool for classifying such textures. We introduce features defined on the estimated parameters of the multispectral random field models and use these features in supervised classification schemes. It is shown that a near perfect classification would be achieved for a database that contains sixteen different color textures.

The advantage of considering color in texture classification is also demonstrated in this work. An equivalent gray-level database is created for the color database used in this study. The gray-level textures are then classified using features derived from graylevel random field models and the performance is compared to that of color textures. There is considerable gain in classification accuracy indicating that color information does provide substantial advantage to the recognition task. 


\section{MULTISPECTRAL RANDOM FIELD MODELS}

Multispectral Random Field Models are the generalization of the gray-level random field models. They were initially developed in [1,2]. These models are capable of characterizing color textures and are able to synthesize a color texture from the estimated parameters of the model fitted to it $[2,1]$. In this work, we utilize three such models for the classification task.

\subsection{Multispectral Simultaneous Autoregressive (MSAR) Model}

The Multispectral Simultaneous Autoregressive (MSAR) model is the first considered model. A pixel location within a two-dimensional $\mathrm{M} \times \mathrm{M}$ lattice is denoted by $\mathbf{s}=(\mathrm{i}, \mathrm{j})$, with $\mathrm{i}, \mathrm{j}$ being integers from the set $\mathrm{J}=\{0,1, \ldots, \mathrm{M}-1\}$. The set of all lattice locations is defined as $\boldsymbol{\Omega}=\{\mathbf{s}=(\mathrm{i}, \mathrm{j}): \mathrm{i}, \mathrm{j} \in \mathrm{J}\}$. The MSAR model relates each lattice position to its neighboring pixels, both within and between image planes, according to the following model equation: $\mathrm{y}_{\mathrm{i}}(\mathbf{s})=\sum_{\mathrm{j}=1}^{\mathrm{P}} \sum_{\mathbf{r} \in \mathrm{N}_{\mathrm{ij}}} \theta_{\mathrm{ij}}(\mathbf{r}) \mathrm{y}_{\mathrm{i}}(\mathbf{s} \oplus \mathbf{r})+\sqrt{\rho_{\mathrm{i}}} \mathrm{w}_{\mathrm{i}}(\mathbf{s}), \quad \mathrm{i}=1 \ldots \mathrm{P}$ with, $y_{i}(\mathbf{s})=$ Pixel value at location $s$ of the $i^{\text {th }}$ plane $\mathbf{s}$ and $\mathbf{r}=$ two dimensional lattices

$\mathrm{P}=$ number of image planes (for color images, $\mathrm{P}=3$, representing: Red, Green, and Blue planes)

$\mathrm{N}_{\mathrm{ij}}=$ neighbor set relating pixels in plane $\mathrm{i}$ to neighbors in plane $\mathrm{j}$ (only interplane

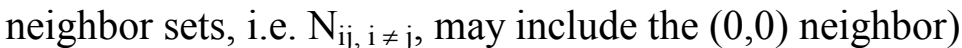

$\theta_{\mathrm{ij}}=$ coefficients which define the dependence of $\mathrm{y}_{\mathrm{i}}(\mathrm{s})$ on the pixels in its neighbor set $\mathrm{N}_{\mathrm{ij}}$

$\rho_{\mathrm{i}}=$ noise variance of image plane $\mathrm{i}$

$\mathrm{w}_{\mathrm{i}}(\mathbf{s})=$ i.i.d. random variables with zero mean and unit variance

$\oplus$ denotes modulo $\mathrm{M}$ addition in each index (a toroidal lattice structure is assumed so a complete neighbor set could be defined for pixels on the boundary of the image)

The image observations are assumed to have zero mean (i.e., the sample mean is computed and subtracted from all pixels).

The parameters associated with the MSAR model are $\boldsymbol{\theta}$ and $\rho$ vectors which collectively characterize the spatial interaction between neighboring pixels within and between color planes. A least-squares (LS) estimate of the MSAR model parameters is obtained by equating the observed pixel values of an image to the expected value of the model equations. This leads to the following estimates $[2,1]$ :

$$
\begin{aligned}
& \hat{\theta}_{\mathrm{i}}=\left[\sum_{\mathbf{s} \in \mathbf{\Omega}} \mathbf{q}_{\mathrm{i}}(\mathbf{s}) \mathbf{q}_{\mathrm{i}}^{\mathrm{T}}(\mathbf{s})\right]^{-1}\left[\sum_{\mathbf{s} \in \mathbf{\Omega}} \mathbf{q}_{\mathrm{i}}(\mathbf{s}) \mathrm{y}_{\mathrm{i}}(\mathbf{s})\right] \text { and } \hat{\rho}_{\mathrm{i}}=\frac{1}{\mathrm{M}^{2}} \sum_{\mathbf{s} \in \mathbf{\Omega}}\left(\mathrm{y}_{\mathrm{i}}(\mathbf{s})-\hat{\boldsymbol{\theta}}_{\mathrm{i}}^{\mathrm{T}} \mathbf{q}_{\mathrm{i}}(\mathbf{s})\right)^{2} \text { where } \\
& \boldsymbol{\theta}_{\mathrm{i}}=\left[\begin{array}{ll}
\boldsymbol{\theta}_{\mathrm{i} 1}^{\mathrm{T}} \mathbf{\theta}_{\mathrm{i} 2}^{\mathrm{T}} \cdots \boldsymbol{\theta}_{\mathrm{iP}}^{\mathrm{T}}
\end{array}\right]^{\mathrm{T}}, \quad \mathbf{q}_{\mathrm{i}}(\mathbf{s})=\left[\mathbf{y}_{\mathrm{i} 1}^{\mathrm{T}}(\mathbf{s}) \mathbf{y}_{\mathrm{i} 2}^{\mathrm{T}}(\mathbf{s}) \cdots \mathbf{y}_{\mathrm{iP}}^{\mathrm{T}}(\mathbf{s})\right]^{\mathrm{T}}, \\
& \mathbf{y}_{\mathrm{ij}}(\mathbf{s})=\operatorname{col}\left\{\mathrm{y}_{\mathrm{j}}(\mathbf{s} \oplus \mathbf{r}): \mathbf{r} \in \mathrm{N}_{\mathrm{ij}}\right\}
\end{aligned}
$$




\subsection{Multispectral Markov Random Field (MMRF) Model}

The second kind of model considered is the Multispectral Markov Random Field (MMRF) Model. A multispectral image may be considered Markovian with respect to its neighbor set if it has the following property:

$\mathrm{p}\left(\mathrm{y}_{\mathrm{i}}(\mathbf{s}) \mid\right.$ all other image observations $)=\mathrm{p}\left(\mathrm{y}_{\mathrm{i}}(\mathbf{s}) \mid\right.$ neighborhood observations $)$

Because the conditional distribution of $\mathrm{y}_{\mathrm{i}}(\mathbf{s})$ given all other observations and $\mathrm{y}_{\mathrm{i}}(\mathbf{s})$ given the neighborhood observations are the same, the best linear estimator of the observed values may be written in terms of the neighborhood observations as:

$\mathrm{y}_{\mathrm{i}}(\mathbf{s})=\sum_{\mathrm{j}=1}^{\mathrm{P}} \sum_{\mathbf{r} \in \mathrm{N}_{\mathrm{ij}}} \theta_{\mathrm{ij}}(\mathbf{r}) \mathrm{y}_{\mathrm{i}}(\mathbf{s} \oplus \mathbf{r})+\mathrm{e}_{\mathrm{i}}(\mathbf{s}), \quad \mathrm{i}=1 \ldots \mathrm{P}$ where the estimation error $\mathrm{e}_{\mathrm{i}}(\mathbf{s})=\sum_{\mathrm{j}=1}^{\mathrm{P}} \sum_{\mathbf{r} \in \mathbf{\Omega}} \mathrm{c}_{\mathrm{ij}}(\mathbf{r}) \mathrm{w}_{\mathrm{j}}(\mathbf{s} \oplus \mathbf{r}), \quad \mathrm{i}=1 \ldots \mathrm{P} \quad$ is a stationary noise sequence with unit variance i.i.d variates, $\mathrm{w}_{\mathrm{i}}(\mathbf{s})$ for some choice of $\mathrm{c}_{\mathrm{ij}}$ 's.

Since the resulting system of equations that could be employed to obtain a LS estimate of the model parameters is nonlinear, an approximate LS estimate approach is employed here. This is an iterative method that involves repeatedly solving the pair of equations [2, 1]: $\hat{\boldsymbol{\theta}}_{\mathrm{n}+1}=\left[\sum_{\mathbf{s} \in \mathbf{\Omega}} \mathbf{Q}_{\mathrm{n}}(\mathbf{s}) \mathbf{Q}_{\mathrm{n}}^{\mathrm{T}}(\mathbf{s})\right]^{-1}\left[\sum_{\mathbf{s} \in \mathbf{\Omega}} \mathbf{Q}_{\mathrm{n}}(\mathbf{s}) \mathbf{y}(\mathbf{s})\right], \quad \mathrm{n}=0,1,2, \ldots$ and

$$
\hat{\boldsymbol{\rho}}_{\mathrm{n}+1}=\left[\hat{\rho}_{1} \hat{\rho}_{2} \hat{\rho}_{3}\right]^{\mathrm{T}}=\frac{1}{\mathrm{M}^{2}} \sum_{\mathbf{s} \in \mathbf{\Omega}}\left[\mathbf{y}(\mathbf{s})-\mathbf{Q}_{\mathrm{n}}^{\mathrm{T}}(\mathbf{s}) \hat{\boldsymbol{\theta}}\right]^{2}, \quad \mathrm{n}=0,1,2, \ldots
$$

where $\mathbf{Q}(\mathbf{s})=\left[\begin{array}{cccccc}\mathbf{y}_{11}^{\mathrm{T}}(\mathbf{s}) & \mathbf{y}_{12}^{\mathrm{T}}(\mathbf{s}) & \mathbf{y}_{13}^{\mathrm{T}}(\mathbf{s}) & \mathbf{0}^{\mathrm{T}} & \mathbf{0}^{\mathrm{T}} & \mathbf{0}^{\mathrm{T}} \\ \mathbf{0}^{\mathrm{T}} & \frac{\rho_{2}}{\rho_{1}} \mathbf{y}_{21}^{\mathrm{T}}(\mathbf{s}) & \mathbf{0}^{\mathrm{T}} & \mathbf{y}_{22}^{\mathrm{T}}(\mathbf{s}) & \mathbf{y}_{23}^{\mathrm{T}}(\mathbf{s}) & \mathbf{0}^{\mathrm{T}} \\ \mathbf{0}^{\mathrm{T}} & \mathbf{0}^{\mathrm{T}} & \frac{\rho_{3}}{\rho_{1}} \mathbf{y}_{31}^{\mathrm{T}}(\mathbf{s}) & \mathbf{0}^{\mathrm{T}} & \frac{\rho_{3}}{\rho_{2}} \mathbf{y}_{32}^{\mathrm{T}}(\mathbf{s}) & \mathbf{y}_{33}^{\mathrm{T}}(\mathbf{s})\end{array}\right]^{\mathrm{T}}$ and

$$
\mathbf{y}_{\mathrm{ij}}(\mathbf{s})=\left\{\begin{array}{rr}
\operatorname{col}\left\{\mathrm{y}_{\mathrm{i}}(\mathbf{s} \oplus \mathbf{r})+\mathrm{y}_{\mathrm{i}}(\mathbf{s} \oplus-\mathbf{r}): \mathbf{r} \in \mathrm{N}_{\mathrm{ii}}\right\}, & \mathrm{i}=\mathrm{j} \\
\operatorname{col}\left\{\mathrm{y}_{\mathrm{j}}(\mathbf{s} \oplus \mathbf{r}): \mathbf{r} \in \mathrm{N}_{\mathrm{ij}}\right\}, & \mathrm{i}<\mathrm{j} \\
\operatorname{col}\left\{\mathrm{y}_{\mathrm{j}}(\mathbf{s} \oplus-\mathbf{r}): \mathbf{r} \in \mathrm{N}_{\mathrm{ji}}\right\}, & \mathrm{i}>\mathrm{j}
\end{array}\right.
$$

$\mathbf{Q}(\mathbf{s})$ is evaluated using $\boldsymbol{\rho}=\hat{\boldsymbol{\rho}}_{n}$ and $\hat{\boldsymbol{\rho}}_{0}$ is taken as $\left[\begin{array}{lll}1 & 1 & 1\end{array}\right]^{T}$. The iteration terminates when convergence is obtained, i.e. subsequent iterations fail to produce significant changes in $\boldsymbol{\theta}$. This approach works well in practice, typically requiring less than 10 iterations to obtain the LS estimate of MMRF model parameters.

\subsection{Pseudo-Markov Random Field (PMRF) Model}

The third model is the Pseudo-Markov Random Field (PMRF) model which has the same model equations as the MMRF: $\mathrm{y}_{\mathrm{i}}(\mathbf{s})=\sum_{\mathrm{j}=1}^{\mathrm{P}} \sum_{\mathbf{r} \in \mathrm{N}_{\mathrm{ij}}} \theta_{\mathrm{ij}}(\mathbf{r}) \mathrm{y}_{\mathrm{j}}(\mathbf{s} \oplus \mathbf{r})+\mathrm{e}_{\mathrm{i}}(\mathbf{s}), \quad \mathrm{i}=1 \ldots \mathrm{P}$ 
However, by restricting the correlation structure of the stationary noise sequences $\left\{\mathrm{e}_{\mathrm{i}}(\mathbf{s})\right\}$ of the PMRF model, it lends itself to a linear LS estimation scheme of its model parameters, instead of the non-linear iterative approach used for the MMRF model. The assumed correlation structure for $\left\{\mathrm{e}_{\mathrm{i}}(\mathbf{s})\right\}$ is:

$$
\mathrm{v}_{\mathrm{ij}}(\mathbf{r})=\mathrm{E}\left\{\mathrm{e}_{\mathrm{i}}(\mathbf{s}) \mathrm{e}_{\mathrm{j}}(\mathbf{s} \oplus \mathbf{r})\right\}=\left\{\begin{array}{c}
-\theta_{\mathrm{ij}}(\mathbf{r}) \sqrt{\rho_{\mathrm{i}} \rho_{\mathrm{j}}}, \quad \mathbf{r} \in \mathrm{N}_{\mathrm{ij}} \\
\rho_{\mathrm{j}}, \quad \mathbf{r}=0 \text { and } \mathrm{i}=\mathrm{j} \\
0, \quad \text { otherwise }
\end{array}\right.
$$

The LS estimate of the PMRF model parameters is given by $[2,1]$ :

$$
\begin{aligned}
& \hat{\boldsymbol{\theta}}=\left[\sum_{\mathbf{s} \in \mathbf{\Omega}} \mathbf{Q}(\mathbf{s}) \mathbf{Q}^{\mathrm{T}}(\mathbf{s})\right]^{-1}\left[\sum_{\mathbf{s} \in \mathbf{\Omega}} \mathbf{Q}(\mathbf{s}) \mathbf{y}(\mathbf{s})\right] \text { and } \hat{\boldsymbol{\rho}}=\left[\hat{\rho}_{1} \hat{\rho}_{2} \hat{\rho}_{3}\right]^{\mathrm{T}}=\frac{1}{\mathrm{M}^{2}} \sum_{\mathbf{s} \in \mathbf{\Omega}}\left[\mathbf{y}(\mathbf{s})-\mathbf{Q}^{\mathrm{T}}(\mathbf{s}) \hat{\boldsymbol{\theta}}\right]^{2} \\
& \text { where: } \mathbf{Q}(\mathbf{s})=\left[\begin{array}{cccccc}
\mathbf{y}_{11}^{\mathrm{T}}(\mathbf{s}) & \mathbf{y}_{12}^{\mathrm{T}}(\mathbf{s}) & \mathbf{y}_{13}^{\mathrm{T}}(\mathbf{s}) & \mathbf{0}^{\mathrm{T}} & \mathbf{0}^{\mathrm{T}} & \mathbf{0}^{\mathrm{T}} \\
\mathbf{0}^{\mathrm{T}} & \mathbf{y}_{21}^{\mathrm{T}}(\mathbf{s}) & \mathbf{0}^{\mathrm{T}} & \mathbf{y}_{22}^{\mathrm{T}}(\mathbf{s}) & \mathbf{y}_{23}^{\mathrm{T}}(\mathbf{s}) & \mathbf{0}^{\mathrm{T}} \\
\mathbf{0}^{\mathrm{T}} & \mathbf{0}^{\mathrm{T}} & \mathbf{y}_{31}^{\mathrm{T}}(\mathbf{s}) & \mathbf{0}^{\mathrm{T}} & \mathbf{y}_{32}^{\mathrm{T}}(\mathbf{s}) & \mathbf{y}_{33}^{\mathrm{T}}(\mathbf{s})
\end{array}\right]^{\mathrm{T}} \text { and } \\
& \mathbf{y}(\mathbf{s})=\left[\begin{array}{lll}
\mathrm{y}_{1}(\mathbf{s}) & \mathrm{y}_{2}(\mathbf{s}) & \mathrm{y}_{3}(\mathbf{s})
\end{array}\right]^{\mathrm{T}}=\mathrm{E}\{\mathbf{y}(\mathbf{s}) \mid \boldsymbol{\theta}\}=\mathbf{Q}^{\mathrm{T}}(\mathbf{s}) \boldsymbol{\theta}, \quad \mathbf{s} \in \boldsymbol{\Omega}
\end{aligned}
$$

\section{DEFINED FEATURES}

The features that are used for classification of color textures are derived from the estimated parameters of the discussed multispectral random field models. In this work, classification of color textures will be compared to classification of gray-level converted versions of the same color textures in order to illustrate the advantage of using color information. As such, in this section both color and grey-level random field model textural features are discussed.

\subsection{Features for Color Textures}

The features for color textures are defined on the estimated $\theta$ and $\rho$ parameters of the considered multispectral model (i.e., MSAR, MMRF, or PMRF). The estimated $\boldsymbol{\theta}$ parameters are used directly whereas ratios of the $\rho$ parameters of different color planes in the form of $\frac{\rho_{\mathrm{r}}}{\rho_{\mathrm{g}}}$ and $\frac{\rho_{\mathrm{r}}}{\rho_{\mathrm{b}}}$ are utilized. The justification for using ratios is to make the features robust to illumination changes. To see this, take a linear imaging model. For such a model, the observed values at each lattice position are assumed to be a product of illumination and spectral reflectance. Under this assumption the $\theta$ 's and ratios of $\rho$ 's are invariant to changes in illumination intensity, i.e., the power of the illumination source changes uniformly across the spectrum. Such a change in illumination will correspond to a linear change in the image observations that is constant across image planes, affecting the mean and variance of the observed image. Changes in the mean vector do not impact the feature set because Random Field model parameters are estimated from mean normalized images. Changes in image variance that are uniform across image planes are reflected in the model parameters as a constant scale factor applied to each $\rho_{\mathrm{i}}$, hence the ratios of $\rho_{\mathrm{i}}$ 's are invariant to such changes. 
The parameter vectors $\theta_{\mathrm{ij}}$ are a function of the selected neighbor sets. For the MSAR model a 4-element unilateral neighbor set is chosen, given by

$$
\mathrm{N}_{\mathrm{ij}}=\{(1,0),(0,1),(1,1),(1,-1)\} \quad \forall \mathrm{i}, \mathrm{j} .
$$

This choice of neighbor set is illustrated in Figure 1 for within a single plane, (i.e., $\mathrm{i}=\mathrm{j}$ ), and it results in a 38 -dimensional feature set, $\mathbf{f}_{\mathrm{MSAR}}$,

$$
\mathbf{f}_{\mathrm{MSAR}}=\left\{\frac{\rho_{\mathrm{r}}}{\rho_{\mathrm{g}}}, \frac{\rho_{\mathrm{r}}}{\rho_{\mathrm{b}}}, \boldsymbol{\theta}_{\mathrm{rr}}, \boldsymbol{\theta}_{\mathrm{rg}}, \boldsymbol{\theta}_{\mathrm{rb}}, \boldsymbol{\theta}_{\mathrm{gr}}, \boldsymbol{\theta}_{\mathrm{gg}}, \boldsymbol{\theta}_{\mathrm{gb}}, \boldsymbol{\theta}_{\mathrm{br}}, \boldsymbol{\theta}_{\mathrm{bg}}, \boldsymbol{\theta}_{\mathrm{bb}}\right\}
$$

with each $\theta_{\mathrm{ij}}$ representing four parameters $\left\{\theta_{1,0}, \theta_{0,1}, \theta_{1,1}, \theta_{1,-1}\right\}$.

The neighbor sets for the MMRF and PMRF models must be symmetric, i.e., $\mathbf{r} \in \mathrm{N}_{\mathrm{ij}}$ $\Leftrightarrow-\mathbf{r} \in \mathrm{N}_{\mathrm{ji}}$. The same four-element neighbor set $\mathrm{N}_{\mathrm{ij}}$ is used for these models. However; the $\theta_{\mathrm{ij}}$ of symmetric neighbors are taken to be equal in these models. Consequently, only half of $\theta_{\mathrm{ij}}$ parameters are used resulting in a 26-dimensional feature vector for each model:

$$
\begin{aligned}
\mathbf{f}_{\mathrm{MMRF}}=\left\{\frac{\rho_{\mathrm{r}}}{\rho_{\mathrm{g}}}, \frac{\rho_{\mathrm{r}}}{\rho_{\mathrm{b}}}, \boldsymbol{\theta}_{\mathrm{rr}}, \boldsymbol{\theta}_{\mathrm{rg}}, \boldsymbol{\theta}_{\mathrm{rb}}, \boldsymbol{\theta}_{\mathrm{gg}}, \boldsymbol{\theta}_{\mathrm{gb}}, \boldsymbol{\theta}_{\mathrm{bb}}\right\} \text { and } \\
\mathbf{f}_{\mathrm{PMRF}}=\left\{\frac{\rho_{\mathrm{r}}}{\rho_{\mathrm{g}}}, \frac{\rho_{\mathrm{r}}}{\rho_{\mathrm{b}}}, \boldsymbol{\theta}_{\mathrm{rr}}, \boldsymbol{\theta}_{\mathrm{rg}}, \boldsymbol{\theta}_{\mathrm{rb}}, \boldsymbol{\theta}_{\mathrm{gg}}, \boldsymbol{\theta}_{\mathrm{gb}}, \boldsymbol{\theta}_{\mathrm{bb}}\right\}
\end{aligned}
$$

It should be noted that the selected four-element neighbor set is not optiml in the sense that it cannot re-generate a visually close image to the original in conjunction with the utilized model. However, as shown later, this compact and rather sub-optimal (from image regeneration point of view) neighbor set can produce excellent classification results.

\subsection{Features for Grey-Level Textures}

The grey-level version of each of the color textures considered in this study is also generated using a conversion method discussed later. The features considered for greylevel texture are also based on random field models. The one plane version $(\mathrm{P}=1)$ of MSAR and MMRF are used for modeling these textures. These models are referred to as SAR and MRF [3, 4]. The LS estimation method is used to estimate $\theta_{i}$ and $\rho$ parameters for these models $[3,4]$. Since there is only one $\rho$ parameter, illumination invariance is obtained by dividing this parameter by the image sample variance, $\hat{\sigma}^{2}$ [5]. Therefore the feature set is: $\mathbf{f}_{\mathrm{SAR}}=\left\{\frac{\rho}{\hat{\sigma}^{2}}, \boldsymbol{\theta}\right\}$ and $\mathbf{f}_{\mathrm{MRF}}=\left\{\frac{\rho}{\hat{\sigma}^{2}}, \boldsymbol{\theta}\right\}$

Various neighbor sets containing from 8 to 80 symmetric neighbors are considered for these models and the ones producing the best results are used in this study. It turns out that the best neighbor sets are a 36 and a 20 element set as shown in Figure 1. These neighbor sets are used for $64 \times 64$ and $16 \times 16$ textures, respectively. The numbers of features associated with these neighbor sets are 19 and 11 for the 36 and 20-element set, respectively, for both the SAR and MRF models. 


\section{CLASSIFICATION METHOD}

A supervised non-parametric nearest-neighbor classifier, along with the leave-oneout error estimation method is used for evaluating the efficacy of the discussed features. In this simple classification scheme, an unknown feature vector $\mathbf{f}^{(t)}$ is assigned to the class associated with its nearest neighbor, as defined by a distance metric $\mathrm{d}(\mathbf{x}, \mathbf{y})$, i.e., assign the unknown image sample to the class $\mathrm{k}$ associated with the training sample for which $\mathrm{d}\left(\mathbf{f}^{(\mathrm{t})}, \mathbf{f}_{\mathrm{i}}^{(\mathrm{k})}\right)$ is minimized, with $\mathbf{f}_{\mathrm{i}}^{(\mathrm{k})}$ representing the ith training sample of class $\mathrm{k}$.

In order to compensate for scale variations and correlations within each feature vector, a normalized distance metric is used $\mathrm{d}\left(\mathbf{f}^{(\mathrm{t})}, \mathbf{f}_{\mathrm{i}}^{(\mathrm{k})}\right)=\sqrt{\left(\mathbf{f}^{(\mathrm{t})}-\mathbf{f}_{\mathrm{i}}^{(\mathrm{k})}\right)^{\mathrm{T}} \hat{\Sigma}_{\mathrm{k}}^{-1}\left(\mathbf{f}^{(\mathrm{t})}-\mathbf{f}_{\mathrm{i}}^{(\mathrm{k})}\right)}$ where the superscript $(\mathrm{t})$ denotes the test sample, and $\hat{\boldsymbol{\Sigma}}_{\mathrm{k}}$ is the sample covariance matrix of the training feature set (including $\mathrm{n}_{\mathrm{k}}$ samples) for class $\mathrm{k}$

$$
\begin{aligned}
& \hat{\boldsymbol{\Sigma}}_{\mathrm{k}}=\frac{1}{\mathrm{n}_{\mathrm{k}}} \sum_{\mathrm{i}=1}^{\mathrm{n}_{\mathrm{kl}}}\left(\mathbf{f}_{\mathrm{i}}^{(\mathrm{k})}-\hat{\mathbf{m}}_{\mathrm{k}}\right)\left(\mathbf{f}_{\mathrm{i}}{ }^{(\mathrm{k})}-\hat{\mathbf{m}}_{\mathrm{k}}\right)^{\mathrm{T}} \text {, with } \hat{\mathbf{m}}_{\mathrm{k}} \text { being the corresponding sample mean } \\
& \hat{\mathbf{m}}_{\mathrm{k}}=\frac{1}{\mathrm{n}_{\mathrm{k}}} \sum_{\mathrm{i}=1}^{\mathrm{n}_{\mathrm{k}}} \mathbf{f}_{\mathrm{i}}^{(\mathrm{k})} \text {. }
\end{aligned}
$$

This normalization method prevents features with large magnitude from dominating the nearest-neighbor distance and compensates for redundancy in the feature set.

\section{IMAGE DATABASES}

A sixteen-class color natural texture database constructed from images available in [6] is used in this study. These are full color Red, Green, and Blue (RGB) images with up to 256 intensity levels for each of the red, green, and blue color components. For each natural texture, two image resolutions are included in the database, $512 \times 512$ and $128 \times 128$. The selected texture images are shown in Figure 2.

Each original image is partitioned into 64 non-overlapping sub-images, providing a total of 1024 images at each selected resolution. These sub-images are $64 \times 64$ and $16 \times 16$ derived from the original $512 \times 512$ and $128 \times 128$ images, respectively.

A second database of gray-level textures is generated from this color database by converting all of the 1024 color images into gray-level ones. This process is carried out using the linear RGB to CIE luminance conversion [7]

$$
\mathrm{Y}_{\mathrm{CIE}}=0.2125 \mathrm{R}+0.7154 \mathrm{G}+0.0721 \mathrm{~B} \text {. }
$$

No re-quantization of the image data was performed and the resultant gray-level images were kept in a floating-point format. Figure $\mathbf{2}$ also illustrates the gray-level converted images.

\section{CLASSIFICATION RESULTS AND CONCLUSIONS}

The leave-one-out classification results are shown in Tables 1 through 4 . In the leave-one-out scheme used here, one sample out of 1024 available samples is taken out and used as the test sample. This left out test sample is then classified using the other 1023 samples as the training set. This procedure is repeated for each of the 1024 samples one at a time resulting in independent classification of each sample. 
Tables 1 and 2 summarize the results for the $64 \times 64$ and $16 \times 16$ images, respectively. The number of correctly classified samples out of the total of 1024 tested samples (tested one at a time) is shown for the color and gray-level images for features derived from different models. Tables $\mathbf{3}$ and $\mathbf{4}$ show the individual classification accuracy rates for each of the sixteen classes.

These results demonstrate that perfect classification can be achieved for $64 \times 64$ color texture images using features of either MSAR or MMRF model. For the PMRF model, there is a single misclassification; one sample from class 12 (Candy Sprinkles) is incorrectly assigned to class 10 (Fabric). For the lower image resolution of $16 \times 16$, classification accuracies remain high at $98.5 \%$ for the MSAR model and $95.9 \%$ for both the MMRF and PMRF models, respectively. The majority of misclassified samples are from class 4 (Leaves), which has textural characteristics that are similar to class 7 (Sand) and class 16 (Grass). Considering the results for both resolutions, it may be concluded that the MSAR based features would be the best choice for the classification task.

As for classification results of the gray-level counterparts images, the accuracy rates for the $64 \times 64$ resolution are high and the SAR and MRF models perform equally well resulting in correct classification rate of $97.8 \%$ and $97.2 \%$, respectively. However, when the resolution is reduced to $16 \times 16$, the performance degrades significantly yielding only $62.1 \%$ and $57.4 \%$ accuracies for the SAR and MRF models, respectively. In this case many of the samples are misclassified and the confusion is spread over almost all classes. As noted before, the structure of the utilized neighbor set is also different for the two resolutions. The 18-element set of Figure 1(a) is used for the $64 \times 64$ case and the 10 -element set of Figure 1(b) is utilized for the $16 \times 16$ resolution. These are neighbor sets that produce the best results from among many that were examined.

By comparing the classification results of color images to their gray-level converted counterparts, the advantage of using color becomes apparent. At the $64 \times 64$ resolution, the color results are clearly better even though an optimal neighbor set is used with the gray-level images. If the same 4-element neighbor set used for color images is utilized for the gray-level images, the accuracy will be much lower. At the $16 \times 16$ resolution, the difference in performance becomes much more pronounced. While the color textures are classified in the high $90 \%$ range, the rate for gray-level images is in the $60 \%$ range. At this resolution, the textural detail within a single plane becomes fuzzy and interaction between different image planes becomes more dominant. The inter-pane interactions, which are somewhat invariant to image resolution, are captured by the multispectral models causing them to perform better than their single plane, gray-level counterparts.

In this work three different multispectral random field models are used for supervised color texture classification. These models capture both inter-plane and intra-plane interactions of image pixels resulting in richer characterization of the image compared to its gray-level counterpart. The performance is tested on 1024 images from a sixteenclass database at two resolutions, $64 \times 64$ and $16 \times 16$. It is shown that a small and compact neighbor set is all that is needed for the classification task. In a leave-one-out performance evaluation scheme and utilizing a normalized nearest-neighbor classifier, perfect classification is obtained for $64 \times 64$ images whereas $16 \times 16$ images produce 
accuracy rates in the $98 \%$ range. It is also concluded that the MSAR model works the best for both image resolutions.

Table 1. Summary of Leave-One-Out Classification Results for $64 \times 64$ Images

\begin{tabular}{|c|c|c|}
\hline Model Type & $\begin{array}{c}\text { No. of Correctly Classified } \\
\text { Out of 1024 }\end{array}$ & $\begin{array}{c}\text { Accuracy } \\
\text { Rate }\end{array}$ \\
\hline MSAR & Color Image Database & $100 \%$ \\
MMRF & 1024 & $100 \%$ \\
PMRF & 1024 & $99.9 \%$ \\
\hline \multicolumn{2}{|c|}{ Gray-Level Image Database } \\
\hline SAR (18-element neighbor set) & 1001 & $97.8 \%$ \\
MRF (18-element neighbor set) & 995 & $97.2 \%$ \\
\hline
\end{tabular}

Table 2. Summary of Leave-One-Out Classification Results for $16 \times 16$ Images

\begin{tabular}{|c|c|c|}
\hline Model Type & $\begin{array}{c}\text { No. of Correctly Classified } \\
\text { Out of 1024 }\end{array}$ & $\begin{array}{c}\text { Accuracy } \\
\text { Rate }\end{array}$ \\
\hline MSAR & Color Image Database & $98.5 \%$ \\
MMRF & 1009 & $95.9 \%$ \\
PMRF & 982 & $95.9 \%$ \\
\hline \multicolumn{2}{|c|}{ Gray-Level Image Database } \\
\hline SAR (10-element neighbor set) & 636 & $62.1 \%$ \\
MRF (10-element neighbor set) & 588 & $57.4 \%$ \\
\hline
\end{tabular}

Table 3. Individual Class Accuracy Rates for $64 \times 64$ Images

\begin{tabular}{|c|c|c|c|c|c|}
\hline \multirow{3}{*}{ Class } & \multicolumn{5}{|c|}{ Accuracy Rate (\%) } \\
\cline { 2 - 6 } & MSAR & MMRF & PMRF & $\begin{array}{c}\text { SAR } \\
\text { (18-neighbor) }\end{array}$ & $\begin{array}{c}\text { MRF } \\
\text { (18-neighbor) }\end{array}$ \\
\cline { 2 - 5 } & & & & 100 & 100 \\
2 & 100 & 100 & 100 & 100 & 100 \\
3 & 100 & 100 & 100 & 98.4 & 100 \\
4 & 100 & 100 & 100 & 95.3 & 87.5 \\
5 & 100 & 100 & 100 & 100 & 100 \\
6 & 100 & 100 & 100 & 100 & 100 \\
7 & 100 & 100 & 100 & 100 & 100 \\
8 & 100 & 100 & 100 & 100 & 100 \\
9 & 100 & 100 & 100 & 98.4 & 96.9 \\
10 & 100 & 100 & 100 & 93.8 & 98.4 \\
11 & 100 & 100 & 100 & 100 & 100 \\
12 & 100 & 100 & 100 & 100 & 98.4 \\
13 & 100 & 100 & 98.4 & 100 & 100 \\
14 & 100 & 100 & 100 & 100 & 78.1 \\
15 & 100 & 100 & 100 & 85.9 & 95.3 \\
16 & 100 & 100 & 100 & 92.2 & \\
\hline
\end{tabular}


Table 4. Individual Class Accuracy Rates for $16 \times 16$ Images

\begin{tabular}{|c|c|c|c|c|c|}
\hline \multirow{3}{*}{ Class } & \multicolumn{5}{|c|}{ Accuracy Rate (\%) } \\
\cline { 2 - 6 } & MSAR & MMRF & PMRF & $\begin{array}{c}\text { SAR } \\
\text { (10-neighbor) }\end{array}$ & $\begin{array}{c}\text { MRF } \\
(10-\text {-neighbor) }\end{array}$ \\
\cline { 2 - 6 } & & & 100 & 84.4 & 90.6 \\
2 & 100 & 100 & 100 & 96.9 & 98.4 \\
3 & 96.9 & 96.9 & 95.3 & 51.6 & 39.1 \\
4 & 100 & 98.4 & 79.7 & 26.6 & 26.6 \\
5 & 84.4 & 75.0 & 93.8 & 57.8 & 42.2 \\
6 & 100 & 100 & 95.3 & 85.9 & 78.1 \\
7 & 100 & 92.2 & 100 & 43.8 & 43.8 \\
8 & 96.9 & 100 & 100 & 39.1 & 34.4 \\
9 & 100 & 100 & 87.5 & 53.1 & 50.0 \\
10 & 100 & 89.1 & 93.8 & 50.0 & 37.5 \\
11 & 98.4 & 90.6 & 100 & 93.8 & 93.8 \\
12 & 100 & 100 & 96.9 & 84.4 & 59.4 \\
13 & 100 & 95.3 & 100 & 39.1 & 48.4 \\
14 & 100 & 100 & 100 & 100 & 96.9 \\
15 & 100 & 100 & 96.9 & 56.3 & 40.6 \\
16 & 100 & 98.4 & 95.3 & 31.3 & 39.1 \\
\hline
\end{tabular}

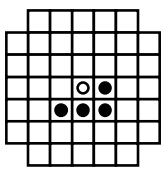

(a) 4

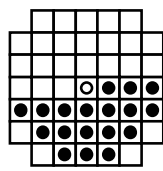

(b)

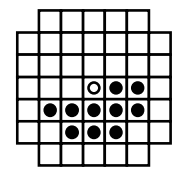

(c)

Figure 1. Neighbor sets used in the image classification experiments. The $(0,0)$ position is represented by the white circles, and the black circles denote the relative neighbor locations.

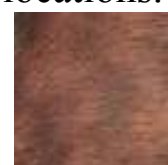

Class 1 (Fur)
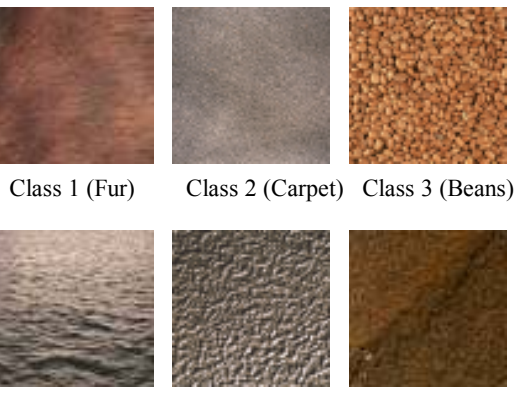
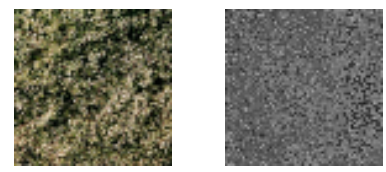

Class 1 (Fur)

Class 4 (Leaves)
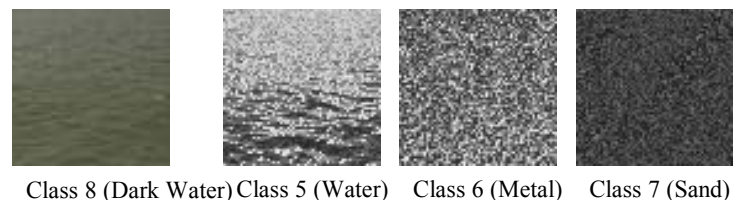

Class 8 (Dark Water) Class 5 (Water)
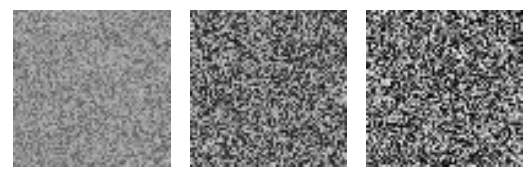

Class 2 (Carpet) Class 3 (Beans) Class 4 (Leaves)
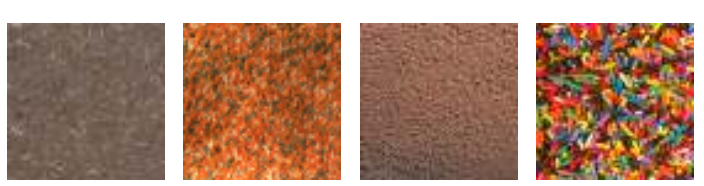

Class 9 (Mulch) Class 10 (Fabric) Class 11 (Coffee) Class 12 (Candy)
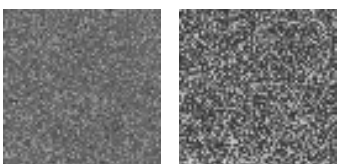

Class 7 (Sand)

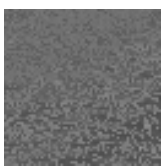

Class 8 (Dark Water)
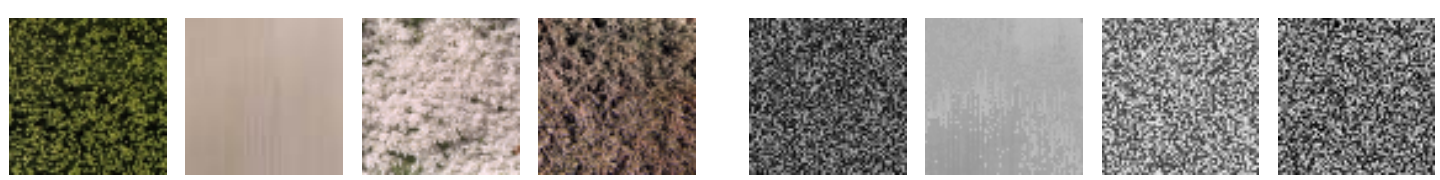

Class 13 (Algae) Class 14 (Wood) Class 15 (Flowers)Class 16 (Grass)

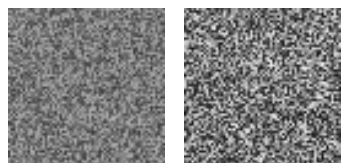

Class 9 (Mulch) Class 10 (Fabric) Class 11 (Coffee) Class 12 (Candy)

Figure 2. Image databases. 


\section{REFERENCES}

1. J. W. Bennett, Modeling and Analysis of Gray Tone, Color, and Multispectral Texture Images by Random Field Models and Their Generalizations, Ph.D. Dissertation, Southern Methodist University, 1997.

2. J. Bennett and A. Khotanzad, Maximum Likelihood Estimation Methods for Multispectral Random Field Image Models, IEEE Trans. on Pattern Analysis and Machine Intelligence, 21, 537-543, June 1999.

3. R. Chellappa and R. L. Kashyap, Synthetic Generation and Estimation in Random Field Models of Images, Proc. IEEE Computer Society Conference on Pattern Recognition and Image Processing, 577-582, Dallas, TX, Aug. 1981.

4. R. L. Kashyap and R. Chellappa, Estimation and Choice Neighbors in SpatialInteraction Models of Images, IEEE Trans. on Information Theory, IT-29, 60-72, 1983.

5. R. Chellappa and S. Chatterjee, Classification of Textures Using Gaussian Markov Random Fields, IEEE Trans. on Acoustics, Speech, and Signal Processing, ASSP-33, 959-963, Aug. 1985.

6. Vision and Modeling Group, MIT Media Laboratory, Vision Texture (VisTex) Database, http://www-white.media.mit.edu/vismod/, 1995.

7. "Basic parameter values for the HDTV standard for the studio and for international programme exchange," Tech. Rep. ITU-R Recommendation BT.709, ITU, 1211 Geneva 20, Switzerland, 1990, formerly CCIR Rec. 709. 